\title{
A COMPARATIVE STUDY OF SEVERITY AND DURATION OF DIABETIC RETINOPATHY IN TYPE 2 DIABETES IN TESSELLATED AND NON-TESSELLATED FUNDUS
} \author{
Justin Thambi Binula Christy6 \\ ${ }_{1}$ Professor, Department of Ophthalmology, Madurai Medical College, Madurai. \\ ${ }^{2}$ Associate Professor, Department of Ophthalmology, Madurai Medical College, Madurai. \\ ${ }_{3}^{3}$ Senior Resident, Department of Ophthalmology, Madurai Medical College, Madurai. \\ ${ }^{4} J u n i o r$ Resident, Department of Ophthalmology, Madurai Medical College, Madurai. \\ 5Junior Resident, Department of Ophthalmology, Madurai Medical College, Madurai. \\ 6Junior Resident, Department of Ophthalmology, Madurai Medical College, Madurai.
}

Ponnusamy Thiyagarajan¹, Natarajan Parvathasundari², Keloth Moidu Febin ${ }^{3}$, Mohandas Deepa ${ }^{4}$, Arumugam Jaga Janani ${ }^{5}$,

\begin{tabular}{l} 
ABSTRACT \\
\hline BACKGROUND \\
Tessellated/tigroid fundus appearance are polygonal dark areas of choroid in between choroidal vessels - due to retinal \\
pigment epithelium atrophy and prominent choroid pigmentation and large surface area along with retinal thinning. Low ocular \\
perfusion pressure associated with thin and or long retinal arterioles reduces the occurrence of Diabetic Retinopathy (DR) \\
compared to non-tessellated fundus.
\end{tabular}

\section{METHODS}

This cross-sectional observational study was conducted among Type 2 Diabetes Mellitus (DM) patients who attended as outpatient and admitted in the wards at Department of Ophthalmology, Govt. Rajaji Hospital, Madurai, to determine whether tessellated fundus is a protective factor for incidence and severity of DR, and to compare the duration and severity of DR in tessellated and non-tessellated fundus. All the patients with DR were examined and assessment of DR done with $+90 \mathrm{D}$ lens under slit lamp microscope. Fundus Fluorescein Angiography (FFA) was performed and grading of DR done as per Early Treatment of Diabetic Retinopathy Study classification (ETDRS) to analyse severity and duration of DR in Type 2 diabetes patients with tessellated and non-tessellated fundus.

\section{RESULTS}

Among the 60 patients, 30 had tessellated fundus and 30 had non-tessellated fundus. The average age group of patients presenting with DR in this study was 45-70 years with male preponderance; 32 patients were myopic, 23 were hyperopic and 5 were emmetrope. On fundus examination, $93.33 \%$ of patients with diabetes for more than 5 years showed majority DR changes. Incidence of Proliferative DR was less in myopes compared to hyperopes; 13 patients had mild non-proliferative diabetic retinopathy (NPDR), 25 had moderate NPDR, 12 had severe NPDR, 2 had very severe NPDR, 5 had early Proliferative Diabetic Retinopathy (PDR) and 3 had high risk PDR. Very severe NPDR, Early PDR and High risk PDR was 100\% absent in tessellated ('p' value was 0.03$)$.

\section{CONCLUSION}

Hence, tessellated fundus is a protective factor for incidence and severity of DR.

\section{KEYWORDS}

Diabetic Retinopathy, Tessellated Fundus, Myopia.

HOW TO CITE THIS ARTICLE: Thiyagarajan P, Parvathasundari N, Febin K M, et al. A comparative study of severity and duration of diabetic retinopathy in type 2 diabetes in tessellated and non-tessellated fundus. J. Evolution Med. Dent. Sci. 2016;5(76):56105612, DOI: $10.14260 /$ jemds/2016/1266

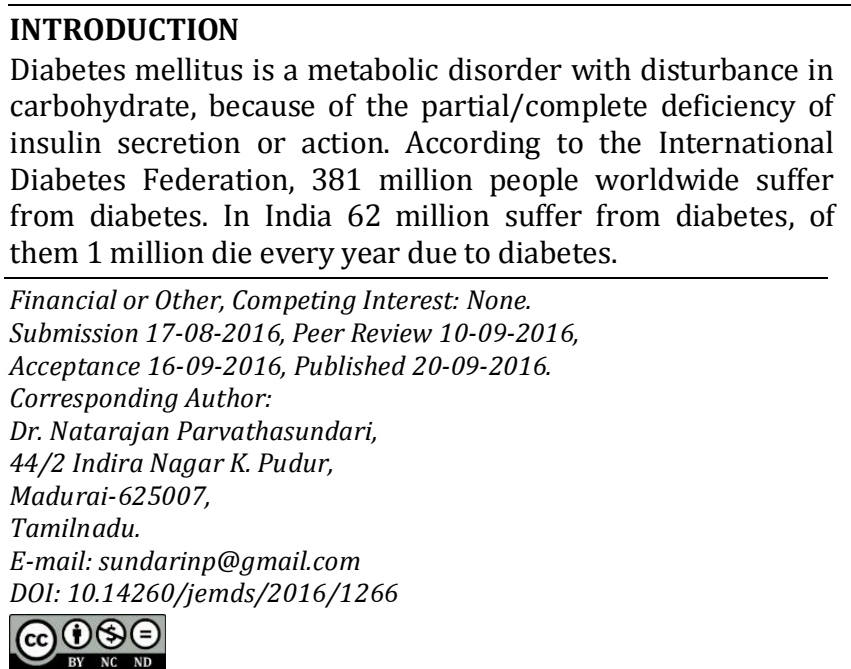

The colour of normal fundus ranges from orange to vermilion. The spectrum of fundus ${ }^{1}$ corresponds to the diffusion of blood, oxyhaemoglobin being the largest factor. Choroidal vasculature also plays an important role in colour of fundus. Tessellated/tigroid fundus ${ }^{2}$ are polygonal dark areas of choroid in between choroidal vessels due to retinal pigment epithelium atrophy and prominent choroid pigmentation. Special features of tessellated fundus include RPE atrophy, choroidal segmentation and large surface area of retinal thinning. ${ }^{3}$

Risk factors for type 2 diabetes include ethnicity, age, lack of physical activity, increased dietary cholesterol intake, obesity with Body Mass Index more than $30 \mathrm{~kg} / \mathrm{sq}$. m, family history, drugs causing diabetes, insulin resistance and smoking. Protective factors from Diabetic Retinopathy (DR) 
include myopia ${ }^{4}$ more than 2.00D, glaucoma, posterior vitreous detachment and retinitis pigmentosa. ${ }^{5}$

\section{METHODOLOGY}

This cross-sectional comparative study was conducted among 60 patients with Type 2 Diabetes Mellitus who attended OPD and wards at Ophthalmology Department, Govt. Rajaji Hospital, Madurai. Subjects with valid consent, who believed to fulfil all eligibility criteria - Patients diagnosed with Type 2 Diabetes, older than 20 years of age with physiological tessellated and non-tessellated fundi and with none of the exclusion criteria - Patients with glaucoma, optic neuropathy, optic atrophy, age related maculopathy, anaemia, renal failure, hypercholesterolemia, hypertension, undergone any ocular surgeries, prior laser treatment and pregnant women were invited to participate in the study.

\section{Ethical Committee Clearance}

Ethical Committee Clearance was obtained from Institutional Review Board/Independent Ethics Committee of Govt. Rajaji Hospital.

\section{Assessment of DR}

Assessment of DR was done for all patients. All the patients with DR were examined with +90 D lens under Slit Lamp Microscope, FFA performed and grading done as per ETDRS classification. ${ }^{6}$ Their refractive status, axial length assessment, fundus photography, etc. was done to divide subjects into 2 groups - tessellated and non-tessellated fundi.

\section{OBSERVATION AND ANALYSIS}

Among 60 patients, 30 patients had tessellated fundus (Fig: 1), 30 patients had non-tessellated fundus. Majority of the patients are between 51 to 70 years of age. The average age group of patients presenting with DR in this study was $45-70$ years with 53\% males and $47 \%$ females. Among the 60 patients 32 were myopes, 23 patients were hyperopes and 5 were emmetrope. Patients with diabetes for more than 5 years showed majority of the Proliferative Diabetic Retinopathy (PDR) or Non-Proliferative Diabetic Retinopathy (NPDR) changes.

Visual Acuity was 6/6 in 11 eyes, $6 / 9$ to $6 / 18$ in 54 eyes and $6 / 24$ to $6 / 60$ in 55 eyes. The defective vision in these patients was due to refractive error, immature cortical cataract and nuclear sclerosis and/or DR changes like clinically significant macular oedema/PDR. On fundus examination 13 patients had mild NPDR, 25 patients had moderate NPDR, 12 patients had severe NPDR, 2 patients had very severe NPDR and 5 patients had early PDR and 3 patients high risk PDR. Very severe NPDR, Early PDR and high risk PDR was $100 \%$ absent in tessellated fundus, but was $100 \%$ present in non-tessellated fundus (Table 1).

Data analysis was done with the help of computer using Epidemiological Information Package - EPI 2010, developed by Centre for Disease Control, Atlanta. Age has a significant correlation with refractive error; ' $p$ ' value 0.0005 . The correlation between tessellated fundus in the severity and duration of DR; 'p' value was 0.03 is also significant. Incidence of proliferative DR was less in myopes when compared to hyperopes; 'p' value was 0.02 .

\begin{tabular}{|c|c|c|c|c|}
\hline \multirow{3}{*}{$\begin{array}{l}\text { Severity of } \\
\text { Retinopathy }\end{array}$} & \multicolumn{4}{|c|}{ Type of Fundus } \\
\hline & \multicolumn{2}{|c|}{$\begin{array}{l}\text { Tessellated } \\
\text { Fundus }\end{array}$} & \multicolumn{2}{|c|}{$\begin{array}{l}\text { Normal } \\
\text { Fundus }\end{array}$} \\
\hline & No. & $\%$ & No. & $\%$ \\
\hline Mild NPDR & 8 & 26 & 5 & 17 \\
\hline Moderate NPDR & 15 & 50 & 10 & 33 \\
\hline Severe NPDR & 7 & 24 & 5 & 17 \\
\hline Very Severe NPDR & 0 & 0 & 2 & 6 \\
\hline Early PDR & 0 & 0 & 5 & 17 \\
\hline High Risk PDR & 0 & 0 & 3 & 10 \\
\hline Total & 30 & 100 & 30 & 100 \\
\hline 'p' value & \multicolumn{4}{|c|}{0.03 Significant } \\
\hline \multicolumn{5}{|c|}{$\begin{array}{c}\text { Table 1: Showing Distribution of Severity of Diabetic } \\
\text { Retinopathy among our Study Subjects Grouped as } \\
\text { With and Without Tessellated Fundus }\end{array}$} \\
\hline
\end{tabular}

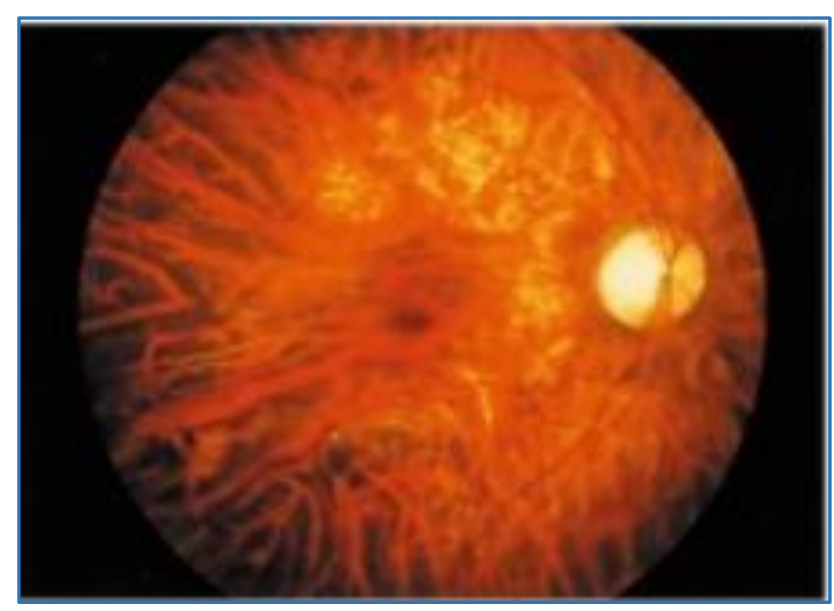

Fig. 1: Tessellated Fundus \& DR

\section{DISCUSSION}

One of the major health problems that have significant impact on socioeconomic life of individual is Diabetes Mellitus. It results in long-term damage, dysfunction and failure of target organs such as eyes, kidneys, nerves, heart and blood vessels. Diabetes mellitus is an endocrine disorder that occurs due to inadequate insulin or insensitivity of cells to insulin, classified as Type 1 IDDM (Insulin Dependent Diabetes Mellitus) or Type 2 NIDDM (Non-Insulin Dependent Diabetes Mellitus) respectively.

Glycaemic control is the fundamental for the management of diabetes as proved by Diabetes Control and Complication Trial (DCCT). ${ }^{7}$ Multiple risk factors that have influence on development of DR such as duration of diabetes, persistent poor glycaemic control, hyperlipidaemia, hypertension, nephropathy, pregnancy anaemia, obesity, genetic factors HLA type - DR3/DR4, intraocular surgeries, etc.

The second major cause for blindness in the world is DR. It has recently got greater attention, because when retinopathy is detected early it is potentially treatable with laser photocoagulation and blindness can be prevented. There is a significant increase in risk of visual loss in patients with delayed diagnosis.

Tessellated/tigroid fundus are dark areas seen in choroid between choroidal vessels due to atrophy of the retinal pigment epithelial layer, retinal layer thinning and prominent choroidal pigmentation. 
Attenuation of arteriolar pressure is seen in patients with tessellated fundus. Pressure attenuation in retinal arterioles is directly proportional to the length and inversely proportional to the diameter of the arteriole segment. A pressure attenuation index ${ }^{8}$ is important in light of the entities and is presumed to protect the retina from DR. The hypothesis is that low end arteriolar pressure ${ }^{9}$ in tessellated fundus due to a low ocular perfusion pressure is a common denominator for the protective factor in DR.

\section{CONCLUSION}

From the above study conducted, it was found that Refractive error has a significant correlation with the age of the patient. Incidence of proliferative DR in tessellated fundus is low compared to non-tessellated/Hyperopic fundus. Very severe NPDR, Early PDR and HR PDR was $100 \%$ absent in tessellated fundus, but was $100 \%$ present in non-tessellated fundus. Incidence of PDR $^{10}$ was less in myopes when compared to hyperopes. Hence, tessellated fundus is a protective factor for the incidence and severity of DR.

\section{REFERENCES}

1. Orihuela-Espina F, Claridge E, Preece SJ. Histological parametric maps of the human ocular fundus: preliminary result. School of Computer Science, University of Birmingham, Edgbaston, B15 2TT, Birmingham, UK 2003

2. Pokharel S, Sherpa D, Shrestha R, et al. Diabetic retinopathy in tessellated fundus. J Nepal Health Res Counc 2014;12(26):49-53.
3. Yoshihara N, Yamashita T, Kii Y, et al. Quantitative analysis of tessellated fundus and its association with choroidal thickness in healthy eyes. Investigative Ophthalmology \& Visual Science 2014;55(13):3591.

4. Lim LS, Lamoureux E, Saw SM, et al. Are myopic eyes less likely to have diabetic retinopathy? Ophthalmology 2010; 117(3):524-30.

5. Arden GB. The absence of diabetic retinopathy in patients with retinitis pigmentosa: implications for pathophysiology and possible treatment. Br J Ophthalmol 2001;85(3):366-70.

6. Lihteh $\mathrm{Wu}$, Fernandez-Loaiza $\mathrm{P}$, Sauma J, et al. Classification of diabetic retinopathy and diabetic macular edema. World J Diabetes 2013;4(6):290-4.

7. Diabetes control and complications trial (DCCT): results of feasibility study. The DCCT research group. Diabetes Care 1987;10(1):1-19.

8. Quigley M, Cohen S. A new pressure attenuation index to evaluate retinal circulation. A link to protective factors in diabetic retinopathy. Arch Ophthalmol 1999;117(1):84-9.

9. Akyol N, Kükner AS, Ozdemir T, et al. Choroidal and retinal blood flow changes in degenerative myopia. Can J Ophthalmol 1996;31(3):113-9.

10. Ganesan S, Raman R, Reddy S, et al. Prevalence of myopia and its association with diabetic retinopathy in subjects with type II diabetes mellitus: a population-based study. Oman J Ophthalmol 2012;5(2):91-96. 